\title{
Decreased conscious level and a renal mass
}

Jolyon Dales, Jenny Prouten, Thrasos Macriyiannis \& Marie-France Kong ${ }^{1}$

Department of Endocrinology, University Hospitals of Leicester NHS Trust, Leicester, UK

University Hospitals of Leicester NHS NHS Trust

\section{Initial Presentation}

- MS, a 70 year-old retired lady found on kitchen floor by her husband having "fits"

- Later observed tonic-clonic seizures

- Past Medical History

Hypothyroidism

Hypertension

Ischaemic Heart Disease

- Drug History

Levothyroxine

Folic acid

Losartan

Simvastatin

Paracetamol

Aspirin

Bendroflumethiazide

Doxazosin

Atenolol

- Blood Pressure was 245/110. She was admitted to the intensive care unit.

- MRI brain showed subcortical oedema with subarachnoid bleed in the left temporal parietal region.
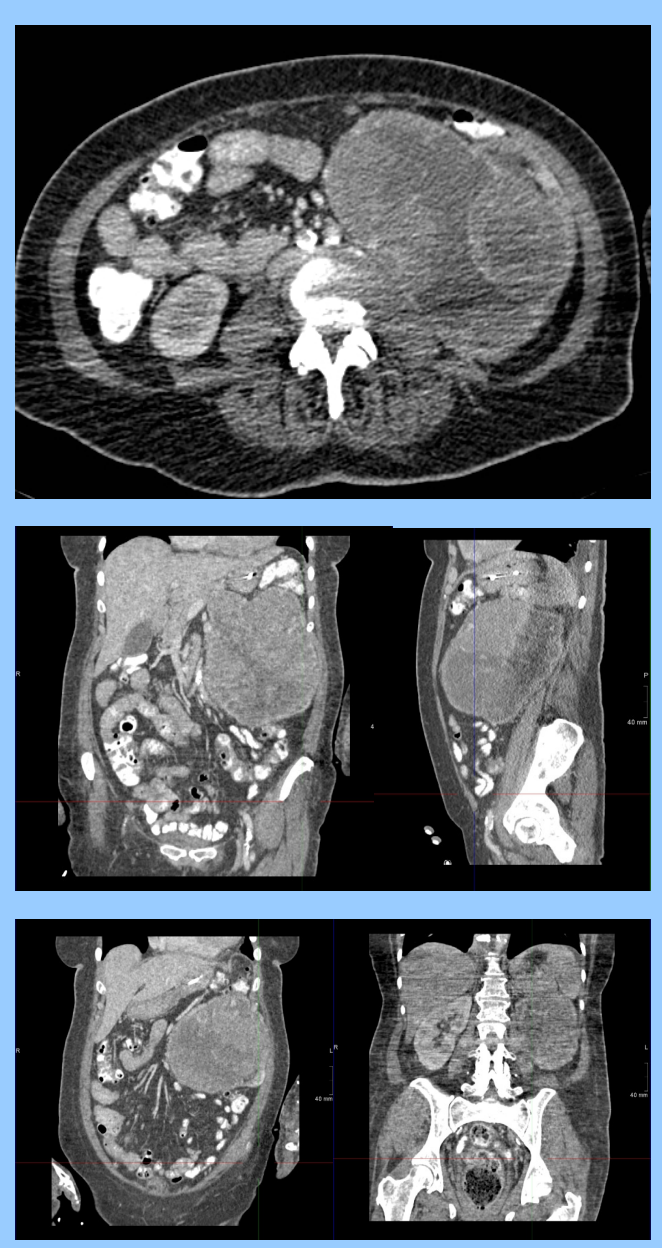

- Investigation for malignant hypertension showed a solid mass measuring $17 \times 17 \times 11 \mathrm{~cm}$ completely replacing the left kidney on CT scan

- She was discharged home on sodium valproate for seizures with a plan for a radical left nephrectomy

\section{Further Progress}

- 2 months later, found unresponsive but no seizure activity was witnessed.

- The paramedic team found her to be hypoglycaemic (near patient blood glucose reading of $1.4 \mathrm{mmol} / \mathrm{l})$

- Two days later, found confused, sweating with incomprehensible speech. Also had haematuria on this occasion.

- Her blood glucose was $1.8 \mathrm{mmol} / \mathrm{l}$. She was admitted for further investigations.

- She experienced spontaneous hypoglycaemia during the night and in the morning and blood samples were taken for insulin and C-peptide levels.

\section{Blood Results}

Laboratory glucose $1.8 \mathrm{mmol} / \mathrm{L}$ (3.3 to 6.0 )

Insulin $\quad<2.0 \mathrm{miu} / \mathrm{L}$ (4.4 to 26 )

C-peptide $\quad<33 \mathrm{pmol} / \mathrm{L}$ (298 to 2350)

Urine Sulphonylureas - NEGATIVE

Further investigations

IGF-I $\quad 6.6 \mathrm{nmol} / \mathrm{L}$ (4.8 to 21.6 )

IGF-II $\quad 105.3 \mathrm{nmol} / \mathrm{L}$

IGF2:IGF1 $16(<10)$

Human Growth Hormone 0.6 ug/L

- This overproduction of IGF2 supports a diagnosis of non-islet cell tumour hypoglycaemia (NICTH)

\section{Resolution}

She was provided with a glucometer and given dietary advice on how to avoid hypoglycaemia

Following left nephrectomy episodes of hypoglycaemia resolved

- No evidence of further disease in patient

\section{Histological Findings}

- Underwent a radical left nephrectomy

Macroscopically the tumour was a large $220 \times 140 \times 100 \mathrm{~mm}$, lobulated, white mass, showing focal necrosis.

Immunohistochemistry shows that the cells are positive for CD99, BCL2 and Vimentin

Suggestive of a solitary fibrous tumour.

Behaviour of extrathoracic solitary fibrous tumours is currently unpredictable, but mostly benign

\section{Previous Case Reports}

Previous case reports have described NonIslet Cell Tumour Hypoglycaemia (NICTH) due to paraneoplastic syndrome predominantly in patients with solid fibrous tumours in the kidney, ${ }^{1-3}$ but also adrenocortical carcinomas, pleural mesotheliomas and in retroperitoneal leiomyosarcomas and hemangiopericytomas. ${ }^{1-3}$

Most case reports describe tumours associated with paraneoplastic IGF-2 production, however NICTH has also been reported to be associated with insulin receptor antibodies, tumour necrosis factor, interleukin 1 or 6 and paraneophlastic production of IGF-1.3

\section{Discussion}

- IGF-II gene located on chromosome 11

- Overproduction of which results in stimulation of the insulin receptors

- NICTH fasting hypoglycaemia and hypoinsulinaemia

- Decreased hepatic glucose production, diminished lipolysis and increased peripheral glucose consumption

- Inappropriately low levels of IGF1, with an increase in IGF2:IGF1 ratio (over 10) suggestive of diagnosis

\section{Treatment \\ - Complete removal of the tumour or reduction of tumour main aspect of treatment}

- Tumour selective chemotherapy or embolisation can have a role where unable to remove tumour ${ }^{2}$

- Glucocorticoids can help by stimulating glycogenolysis and gluconeogenesis and can help shrinkage of tumour ${ }^{2}$

- Short term beneficial effect can be achieved with parenteral glucose or dietary guidelines ${ }^{1}$

- Somatostatin analogues do not help as somatostatin receptors in tumour likely non functional

\section{Summary}

- NICTH is a rare but serious complication of malignancy and should be considered in patient with recurrent hypoglycaemia and hypoinsulinaemia 